\title{
Trauer um Prof. Dr. J. H. Holzner - ein Leben für die Pathologie
}

\author{
Roland Sedivy
}

Eingegangen: 12. Mai 2013 / Angenommen: 14. Mai 2013 / Online publiziert: 18. Juni 2013

(C) Springer-Verlag Wien 2013

Kaum ist es her, dass wir am 12.12.12 gemeinsam mit dem 9. Nachfolger am Rokitansky Lehrstuhl für Pathologie, Johann Heinrich Holzner (1924-2013), 200 Jahre Restitution der Wiener Pathologie feierten, erreicht uns die Nachricht seines Todes. Holzner wurde am 3.12.1924 in Bregenz geboren, studierte Medizin in Innsbruck und in Wien. Er promovierte 1951 und begann im selben Jahr unter Chiari seine Facharztausbildung. Von 1958-1959 war Holzner Research Fellow auf der Pathologie des Mt. Sinai Hospitals in New York bei Hans Popper, verbrachte wissenschaftliche Aufenthalte am Mallory Institute in Boston und am NIH in Bethesda. Nach seiner Rückkehr wurde Prof. Holzner 1963 Leiter des histologisch-zytologischen Labors der II. Univ.-Frauenklinik in Wien. Seine Habilitation erfolgte im Jahr 1966. Schließlich wurde er 1969 zum Nachfolger von Chiari berufen. Holzner fand ein Institut im schlechten baulichen Zustand vor. Mit Zähigkeit und Geduld gelang es ihm, die Renovierung stückweise gegenüber einer trägen, wohl auch desinteressierten Verwaltung durchzusetzen. Holzner war einer der ersten Pathologen, die ihr Wissen um neue Entwicklungen aus Amerika holten, wodurch sein Labor rasch den Ruf eines interessanten und technisch innovativen Arbeitsplatzes bekam. Forschungsbereiche wie jene der Elektronenmikroskopie, Hormon- sowie Zytodiagnostik, Zytogenetik und Histochemie wurden von Holzner etabliert, womit seine Ära für den Aufbruch der Pathologie in die Moderne steht. Prof. Holzner genoss internationalen Ruf und hohes Ansehen. Er war unter anderem Präsident der European Society of Pathology und der Deutschen Gesellschaft für Pathologie, sowie Tagungspräsident des World Congress der IAP 1986 in Wien. 1991 erfolgte die Übersiedlung des Institutes aus den traditionsreichen Räumen des alten Hauses in das neue AKH. Prof. Holzner hat auch den Autor am Institut in Wien aufgenommen. Ich habe ihn als Lehrer und Professor der alten Schule kennengelernt, der jeden Morgen über 48 Semester hindurch seine Vorlesung pünktlich hielt, Musterobduktionen abwickelte und eigentlich immer ein Lächeln auf den Lippen hatte. Seine Lehrbücher und das Rigorosum bei ihm waren und sind Generationen von Ärzten gut als echter Prüfstein in Erinnerung - er war eine Institution, ein Grand Senior der Pathologie des 20. Jahrhunderts. Österreichs Pathologie hat einen ihrer Großen verloren. Unser inniges Mitgefühl gilt seinen Hinterbliebenen.

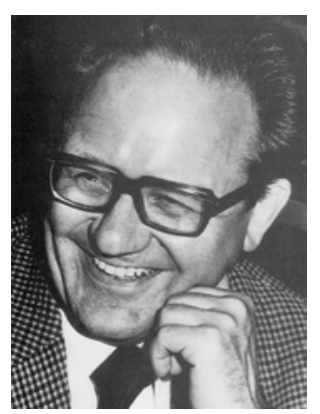

Johann Heinrich Holzner (19242013), Vorstand von 1969-1993

Prim. Univ.-Prof. Dr. R. Sedivy, MD PhD MLS $(\bowtie)$

Institut für Pathologie, LK St. Pölten, Propst Führer Str. 3-4,

3100 St. Pölten, Österreich

E-Mail: roland.sedivy@meduniwien.ac.at

Prim. Univ.-Prof. Dr. R. Sedivy, MD PhD MLS

Zentrum für Pathologie, Danube Private University, 3500

Krems/Donau, Österreich 